\title{
Uncovering the neurochemistry of reward and aversiveness
}

\author{
Carmelo M. Vicario* \\ School of Psychology, University of Queensland, Brisbane, QLD, Australia \\ *Correspondence: carmelo.vicario@uniroma1.it; c.vicario@uq.edu.au \\ Edited by: \\ Nicola Maggio, The Chaim Sheba Medical Center, Israel \\ Reviewed by: \\ Geraldine A. Wright, Newcastle University, UK \\ Christopher D. Fiorillo, Korean Advanced Institute of Science and Engineering, South Korea
}

Keywords: dopamine, serotonin, reward, aversiveness, neurochemistry

\section{A commentary on}

Two dimensions of value: dopamine neurons represent reward but not aversiveness

by Fiorillo, C. D. (2013). Science 341

546-549. doi: 10.1126/science.1238699

In a recent issue of Science was published an interesting report regarding the role of dopaminergic neurons on reward and aversiveness (Fiorillo, 2013). In contrast to what is claimed by some physiological and computational models (e.g., Schultz et al., 1997), the author provides a number of arguments to support the suggestion that reward and punishment have to be considered as two distinct neural dimensions. In particular, the author focuses on the evidence that dopaminergic neurons are selectively activated by rewarding outcomes, while these same neurons are insensitive to aversiveness. Thus, the author concludes a selective role for dopamine in detecting reward, and suggests that other (not dopaminergic) neurons should be sensitive to aversiveness. Recent insights suggest that these neurons may be serotonergic. For example, Limebeer et al. (2004) have shown that depletion of forebrain serotonin (5-HT) by 5,7 dihydroxytryptamine (5,7-DHT) lesions prevents induced conditioned disgust reactions such as "gaping," the predominant conditioned rejection reaction (Parker, 2003) to "tasterelated" aversive stimulation. Wright et al. (2010) have shown that learning to avoid odors associated with the malaise caused by ingesting toxins is mediated by serotonin. Moreover, it was recently demonstrated that partial depletion of 5-HT, in the insular cortex, prevents induced conditioned disgust reactions (Tuerke et al., 2012; see also Vicario, 2013, in press for a recent discussion about this issue). Finally, there is evidence of altered serotoninergic activity in Anorexia Nervosa (AN) (e.g., Jean et al., 2012), a psychiatric disorder characterized by a marked aversive sensitivity for food (Vicario, 2013, in press).

The works mentioned above support the "two dimensions" hypothesis (Fiorillo, 2013) for reward and aversiveness. In particular, it is suggested that serotoninergic neurons could be the principal candidate of aversiveness processing.

The evidence provided by these studies, which are based on the behavioral effects of block (removal) of serotonin, are difficult to interpret given the relevant number of variables that remains out of control. Thus, the definitive evidence could come from experiments that monitor activity of serotonin neurons on a fast timescale and relate it to reward and aversiveness in carefully controlled experiments. Moreover, future works might wish to investigate the existence of "ON" or "OFF" operating mechanism also for serotonin. In fact, if serotonin is the key neurotransmitter underlying aversiveness, there may be some serotonin neurons that signal aversive-ON (punishment) and others that signal aversive-OFF (absence of punishment and "negative reinforcement").

\section{ACKNOWLEDGMENTS}

I would like to thank Nicholas Ashton for his help in the checking of English spelling and the Reviewer Christopher D. Fiorillo for the cogent and helpful comments.

\section{REFERENCES}

Fiorillo, C. D. (2013). Two dimensions of value: dopamine neurons represent reward but not aversiveness. Science 341, 546-549. doi: 10.1126/science.1238699

Jean, A., Laurent, L., Bockaert, J., Charnay, Y., Dusticier, N., Nieoullon, A. et al. (2012). The nucleus accumbens 5-HTR4-CART pathway ties anorexia to hyperactivity. Transl. Psychiatry 2 , e2038. doi: 10.1038/tp.2012.131

Limebeer, C. L., Parker, L. A., and Fletcher P. J. (2004). 5, 7-dihydroxytryptamine lesions of the dorsal and median raphe nuclei interfere with lithiuminduced conditioned gaping, but not conditioned taste avoidance, in rats. Behav. Neurosci. 118, 1391-1399. doi: 10.1037/0735-7044.118.6.1391

Parker, L. A. (2003) Taste avoidance and taste aversion: evidence for two independent processes. Learn. Behav. 31,165-172. doi: 10.3758/BF03195979

Schultz, W., Dayan, P., and Montague, P. R. (1997). A neural substrate of prediction and reward. Science 275, 1593-1599. doi: 10.1126/science.275. 5306.1593

Tuerke, K. J., Limebeer, C. L., Fletcher, P. J., and Parker, L. A. (2012). Double dissociation between regulation of conditioned disgust and taste avoidance by serotonin availability at the $5-\mathrm{HT} 3$ receptor in the posterior and anterior insular cortex. J. Neurosci. 32, 13709-13717. doi: 10.1523/JNEUROSCI.204212.2012

Vicario, C. M. (2013). Inborn mechanisms of food preference and avoidance: the role of polymorphisms in neuromodulatory systems. Front. Mol. Neurosci. 6:16. doi: 10.3389/fnmol.2013.00016

Vicario, C. M. (in press). Alterated insula response to sweet taste processing in recovered anorexia and bulimia nervosa: a matter of disgust sensitivity? Am. J. Psychiatry doi: 10.1176/appi.ajp.2013. 13060748

Wright, G. A., Mustard, J. A., Simcock, N., RossTaylor, A. A. R., McNicholas, L. D., Popescu, A. (2010). Parallel reinforcement pathways for conditioned food aversions in the honeybee. Curr. Biol. 20, 2234-2240. doi: 10.1016/j.cub.2010.11.040

Received: 02 October 2013; accepted: 01 November 2013; published online: 19 November 2013.

Citation: Vicario CM (2013) Uncovering the neurochemistry of reward and aversiveness. Front. Mol. Neurosci. 6:41. doi: 10.3389/fnmol.2013.00041

This article was submitted to the journal Frontiers in Molecular Neuroscience.

Copyright $(\odot) 2013$ Vicario. This is an open-access article distributed under the terms of the Creative Commons Attribution License (CC BY). The use, distribution or reproduction in other forums is permitted, provided the original author(s) or licensor are credited and that the original publication in this journal is cited, in accordance with accepted academic practice. No use, distribution or reproduction is permitted which does not comply with these terms. 